\title{
Die Enteignungen in Simbabwe aus völkerrechtlicher Sicht
}

Von Wolf Peterhoff, Frankfurt/M.

Die ethnopolitischen Konflikte nach Ende der Blockkonfrontation stellen auch und insbesondere in Afrika die Frage nach den effektiven Möglichkeiten und der Reichweite des Völkerrechts ${ }^{1}$. Einem dieser Komplexe ist der vorliegende Aufsatz gewidmet.

\section{I. Überblick}

Nach jahrzehntelanger Apartheid im ehemaligen britischen Rhodesien wurde 1979 Josiah Gumede nach den - vornehmlich durch britische und US-amerikanische Vermittlung zustande gekommenen - ersten allgemeinen und gleichen Wahlen erster schwarzafrikanischer Präsident von Simbabwe. Am 18. April 1980 erlangte Simbabwe unter Robert Mugabe die Unabhängigkeit und wurde im August Mitglied der Vereinten Nationen ${ }^{2}$. Seit dem Jahr 1987 sind aufgrund der Abschaffung von bis dato für jene reservierten Parlamentssitzen Angehörige der weißen Bevölkerung nicht mehr im Parlament vertreten. Am 16. März 1992 wurde ein Gesetz über die Landenteignung („Land Acquisition Bill“) beschlossen, auf dessen Grundlage $50 \%$ des Bodens der weißen Farmer gegen Entschädigung zur Enteignung freigegeben wurde ${ }^{3}$. Zu diesem Zeitpunkt lebten noch 90.000 Weiße im Land. Entsprechend der so genannten „Landreform“ - nach dem Landerwerbsgesetz der simbabwischen Regierung aus dem Jahre 2000 unter Präsident Robert Mugabe - werden bis heute landwirtschaftliche Großbetriebe von mehr als 2900 weißen Farmern ohne Entschädigung enteignet und das eingezogene Land an schwarze Afrikaner vergeben ${ }^{4}$; diese bewirtschaften fortan jedoch ausnahmslos kleine Parzellen von zuvor zusammenhängendem Land. Die Konfiskation, im Sinne einer entschädigungslosen Enteignung, erfolgt

Heintze, Hans-Joachim, Entwickeln sich allgemeine völkerrechtliche Regeln zum Minderheitenschutz? Anmerkungen zu den Lund-Empfehlungen eines internationalen Expertenteams über Mitwirkungsrechte von Minderheiten, in: HuV 2000, S. 89 ff.

2 Land, Housing and Property Rights in Simbabwe, S. 10; Madhuku, Lovemore, The Impact of the European Court of Human Rights in Africa, in: AJICL 1996, S. 932 ff. (933).

$3 \mathrm{Zu}$ diesem Zeitpunkt besaßen die weißen Farmer $39 \%$ des Landes, vgl. Land, Housing and Property Rights in Simbabwe, S. 12.

4

FAZ v. 25.06.02. 
aufgrund von $\S 5$ und $\S 8$ des Landerwerbsgesetzes ${ }^{5}$. Dabei geschieht die Vertreibung der weißen Farmer von ihrem Eigentum unter Androhung oder Ausführung unmittelbaren staatlichen Zwangs ${ }^{6}$. In anderen Fällen unterlassen Polizeikräfte das gebotene Einschreiten $^{7}$, sofern die Farmbesetzungen durch sogenannte „Kriegsveteranen“ durchgeführt werden. Aufgrund eines präsidialen Dekretes vom 10. Mai 2002 ist der Rechtsweg gegen die nach $\S 5$ und $\S 8$ vorgenommenen Enteignungen nicht mehr offen. Darüber hinaus wurden von der Regierung Mugabe unzufriedene Anhänger, die so genannten „Kriegsveteranen“, ermutigt, wilde Besetzungen und Enteignungen, zuweilen unter Einschluss von Plünderungen und Gewalt, zu beginnen ${ }^{8}$.

\section{Besonderheiten der völkerrechtlichen Beurteilung}

Die vorliegend in Rede stehenden Enteignungen treffen mindestens zum Teil eine Gruppe von Staatsbürgern des enteignenden Staates. Insofern ist - anders etwa als bei in solcher Hinsicht „klaren“ Fällen - besonders zu untersuchen, ob sich die weißen Farmer überhaupt unter den Begriff „Volksgruppe“ subsumieren lassen. In diesem Kontext ist hier besonders zu prüfen, ob schon das subjektive Element des Zugehörigkeitsempfindens zum Vorhandensein einer Volksgruppe führt. In diesem Fall könnten den betroffenen Alteigentümern bereits eigene Gruppenrechte zukommen. Des weiteren ist zu untersuchen, ob bei dem Betroffensein von inländischen Staatsangehörigen die Regeln des Völkerrechts zur Anwendung kommen.

Neben den möglichen Restitutions- oder Entschädigungsansprüchen der Alteigentümer ist sodann noch der Frage nachzugehen, ob und von wem hier diplomatischer Schutz zugunsten der Enteigneten ausgeübt werden kann. Im Rahmen der Prüfung der Klage- und Rechtsschutzaussichten wird neben den in Frage kommenden Verfahren vor nationalen Gerichten und dem IGH eine Klage vor dem Afrikanischen Gerichtshof für Menschenrechte ${ }^{9}$ wegen eines Verstoßes gegen die Afrikanische Charta der Menschenrechte und Rechte der Völker $\mathrm{zu}$ untersuchen sein.

FAZ v. 15.08.02.

7 Vgl. Commissioner of Police vs. Commercial Farmer's Union 2000 (1) ZLR 503 (H).

8 Vgl. dazu Schwarz, Birgit, Spiel mit der Hoffnung, in: Der Spiegel, 35/2002, S. 120.

9 Vgl. dazu nur die GV-Res. 3212 (XXIX) (1974), op. § 1; Res. 33/15 (1978), Präambel § 71; Res. 34/30 (1979), Präambel § 101. 


\section{Bestehen einer Volksgruppe}

Ein naheliegendes objektives Abgrenzungskriterium der hauptsächlich betroffenen Landeigentümer zur nicht betroffenen Mehrheit in Simbabwe ist im vorliegenden Fall die unterschiedliche Hautfarbe. Allerdings soll in Simbabwe nach dem Ende der Dominanz der weißen Bevölkerung die Anknüpfung an „Rasse“ eben nicht mehr - als Form einer ,umgekehrten" Apartheid - stattfinden ${ }^{10}$. Letztlich kommt es zur Herausarbeitung objektiver Anknüpfungspunkte hier dennoch nur darauf an, wie auch immer geartete äußerliche Unterscheidungsmerkmale aufzufinden. Viel spricht dafür, den - auch in völkerrechtlichen Abkommen genutzten - Begriff ,race“"11, der wohl letztlich auch eine Unterscheidbarkeit aus soziologischer Sicht enthält ${ }^{12}$, nur im Sinne einer allgemein ethnischen Unterscheidbarkeit der Gruppen zu verstehen ${ }^{13}$. So wäre dann auch bei der weißen Bevölkerung Simbabwes eine gemeinsame ethnische Identität entsprechend Art. $27 \mathrm{IPbpR}$ festzustellen. Daneben kommen aber auch gemeinsame religiöse, kulturelle, geschichtliche und sprachliche Merkmale neben der Wahrnehmung der subjektiven Gruppenzugehörigkeit in Betracht ${ }^{14}$. Dies alles unterscheidet die Gruppe von der Majorität der schwarzen Simbabwer

\section{Die Enteignungen der weißen Farmer}

In Simbabwe haben im Zuge der „Landreform“ aus dem Blickwinkel des Völkerrechts zum Teil ,wilde“ Enteignungen stattgefunden, welche allerdings von der Regierung begrüßt und gefördert wurden. „Wilde“ Enteignungen sind Plünderungen, die aber zumindest mit Billigung der Hoheitsgewalt stattfinden ${ }^{15}$. Die hier in Rede stehenden Handlungen sind damit gemäß allgemeinen völkerrechtlichen Grundsätzen - dem simbabwischen Staat zurechen$\operatorname{bar}^{16}$. Als völkerrechtswidrige Konfiskationen kommen insbesondere die entschädigungslo-

Duchrow, Julia, Völkerrechtlicher Minderheitenschutz in einem multikulturellen Staat? Das Beispiel der weißen Bevölkerung Südafrikas, S. 30.

11 So z. B. in Art. 1 (3) SVN; Art. 2 (1) der AEMR; Art. 2 (1) IPbpR.

12

Denn die Angehörigen einer Minderheit sind ja immer diejenigen, die von anderen als solche betrachtet werden.

13

14

15

Banz, Michael, Völkerrechtlicher Eigentumsschutz durch Investitionsschutzabkommen, 1987, S. 164.

16

Vgl. dazu die Rechtsprechung des IGH zum Teheraner Geiselfall. 
sen Enteignungsmaßnahmen gemäß $\S 5$ sowie $\S 8$ des Landerwerbsgesetzes in Betracht ${ }^{17}$. So haben seit dem Februar des Jahres 2000 über 3000 Farmer ihr Land verlassen müssen ${ }^{18}$.

\section{Die völkerrechtliche Beurteilung der Konfiskationen}

\section{Anwendung völkerrechtlichen Fremdenrechts}

Dem Eingriff in das Eigentum von Ausländern wird zu seiner möglichen Rechtfertigung vorausgesetzt, dass er zum öffentlichen Wohl geschieht. Im übrigen besteht die völkerrechtliche Notwendigkeit, ein faires Verfahren und gerichtlichen Schutz zu gewährleisten $^{19}$. Gerade dies ist aber bezüglich der enteigneten Farmer zweifelhaft. In der Tat wurde ihnen der Rechtsweg mittlerweile durch das präsidiale Dekret vom 10. Mai 2002 versagt. Für den Fall, dass die weißen Farmer nicht Staatsangehörige von Simbabwe sind, sind die fremdenrechtlichen Regelungen ohne weiteres anwendbar. Die Anforderungen des fremdenrechtlichen Mindeststandards wurden durch die ohne Rechtsschutz und Entschädigung erfolgten Enteignungen allerdings nicht eingehalten, so dass für diesen Fall das Fremdenrecht verletzt ist.

Fraglich ist jedoch, ob völkerrechtliche Schutznormen auch für diejenigen Alteigentümer anwendbar sind, die ausschließlich die simbabwische Staatsangehörigkeit besitzen. In diesem Fall könnte nämlich entsprechend der Act of State-Doktrin die Enteignungszuständigkeit Simbabwes ohne Einschränkungen gegeben sein. Allerdings könnte bei einer Überlagerung des hergekommenen Fremdenrechts durch das völkerrechtliche Anliegen des Schutzes der Menschenrechte auch für eine Anwendung der Regeln auf Inländer zu plädieren sein. Wie bereits oben im Allgemeinen Teil festgestellt, kann dies jedoch nicht unbeschränkt gelten. Andernfalls würde jede domaine réservée beschnitten. Da es sich bei dem Recht auf Achtung des Privateigentums nicht um eines der herausragenden Menschenrechte handelt, ist die Anwendung der Fremdenrechtsnormen zum Eigentumsschutz auf Inländer in Simbabwe abzulehnen.

Dies ist auch die Auffassung des eidgenössischen Bundesrats, Interpellation 02.3436, v. 17.09.2002.

18

Vgl. Simbabwe in black and white, The Washington Times, Editorial, 26.08.2002.

19

Seidl-Hohenveldern, Ignaz, in: Neuhold, Hanspeter / Hummer, Waldemar / Schreuer, Christoph (Hrsg.), Österreichisches Handbuch des Völkerrechts, Bd. 1, 1997, Rn. 1022. 


\section{Diskriminierende Enteignung}

Diskriminierende Enteignungen sind dann gegeben, wenn Vermögen der weißen Volksgruppe enteignet, das der restlichen Bevölkerung hingegen von diesen Maßnahmen verschont wird. Da in Simbabwe von den bislang durchgeführten Maßnahmen ausnahmslos das Eigentum der Angehörigen der weißen Volksgruppe betroffen ist, handelt es sich um eine völkerrechtswidrige diskriminierende Enteignung. Rechtfertigungsgründe für die kollektive Enteignung sind nicht ersichtlich.

\section{Vertreibung}

In Betracht kommt weiter die Völkerrechtswidrigkeit der mit den Konfiskationen einhergehenden Vertreibung der weißen Farmer sowie die Verwehrung des Rückkehrrechts in die Heimat. Die Maßnahmen aufgrund der $\S 5$ und $\S 8$ des Landerwerbsgesetzes begannen im Jahre $2000^{20}$. Die Handlungen wurden zum Teil unter der Anwendung von Zwang vorgenommen, so dass der Tatbestand der Vertreibung erfüllt ist. Teilweise verließen die weißen Farmer ihre Heimat allerdings auch aufgrund von Drohungen oder anders ausgeübtem Druck. In diesem Fall ist terminologisch „Flucht“ anzunehmen, die gleichwohl wie „Vertreibung“" zu bewerten ist.

\section{Völkerrechtswidrigkeit der Vertreibung nach Völkervertragsrecht}

Die Handlungen sind am positiven Völkerrecht zu messen. Die Regelungen der HLKO sind nicht einschlägig, da die Maßnahmen außerhalb eines bewaffneten internationalen Konflikts stattgefunden haben; die bestehenden inneren Konflikte in Simbabwe können den Schutzbereich nicht ausdehnen. Verstöße gegen andere Menschenrechtsverträge sind nicht ersichtlich. Zwar ist im Völkermordverbot auch ein Verbot der Vertreibung enthalten. Jedoch sind die strengen Voraussetzungen des Völkermordtatbestandes durch die hier in Rede stehenden Handlungen nicht erfüllt.

$\mathrm{Zu}$ überlegen ist, ob die Vertreibungshandlungen möglicherweise mit dem völkerrechtlichen Recht auf Ausweisung vereinbar sind. Auf dieses könnte dann gegebenenfalls die Regierung Simbabwes rekurrieren. Das könnte allerdings nur für jene Weißen gelten, welche nicht Staatsangehörige Simbabwes sind. Allerdings gilt das Recht auf Ausweisung, wie bereits gezeigt, ohnehin nicht für eine Massenausweisung. Es ist jedoch zweifelhaft, ob es sich um eine solche handelt. Viele vertriebene Weiße sind aus dem Land geflohen. Ein behördlicher Ausweisungsbefehl gegenüber den Angehörigen der weißen Volksgruppe 
liegt, im Unterschied zur Vertreibung von den Farmen, jedoch nicht vor. Eine Berufung auf das Recht auf Ausweisung ist nicht möglich.

\section{Völkerrechtswidrigkeit der Vertreibung nach Völkergewohnheitsrecht}

Das Völkergewohnheitsrecht verbietet die Aufhebung der Verbindung zwischen Bevölkerung und angestammtem Siedlungsgebiet beziehungsweise erlaubt diese nur bei einem freiwilligem Abwanderungsentschluss der Bevölkerung ${ }^{21}$. Hier könnte fraglich sein, ob dies auch dann gilt, wenn die betreffende - eigene - Bevölkerung nicht außer Landes, sondern „,nur“ von ihrem Land vertrieben wird. In diesem Fall werden also eigene Staatsangehörige innerhalb ihres Heimatstaates vertrieben. Entsprechend den mittlerweile zu Recht erstarkten Grundsätzen des Rechts auf die Heimat ist die Bindung der Bevölkerung primär gegenüber der konkreten Heimat anzunehmen. Sie ist nicht an ein spezielles Staatsgebiet geknüpft. „Vertreibung“ kann daher auch innerstaatlich stattfinden und eigene Staatsangehörige betreffen. Die Vertreibungshandlungen gegenüber den weißen Farmern in Simbabwe sind mithin, entsprechend den hier vertretenen völkerrechtlichen Grundsätzen, ohne weiteres rechtswidrig ${ }^{22}$.

\section{Recht auf die Heimat der weißen Farmer}

Für die Anwendbarkeit des in der europäischen Nachkriegsgeschichte geprägten Begriffs des „Rechts auf die Heimat““23 müsste die Volksgruppe auf dem in Rede stehenden Gebiet ansässig sein. Die weiße Bevölkerung in Simbabwe ist, ebenso wie die schwarze Majorität, dort seit Beginn des 19. Jahrhunderts ansässig. Ihr steht somit das Recht auf die Heimat zu. Mit der erfolgten Vertreibung wandelt sich das Recht auf die Heimat zu einem Recht auf Rückkehr in die angestammte Heimat. Das aus dem völkerrechtlichen Selbstbestimmungsrecht abzuleitende Recht auf die Heimat muss der weißen Volksgruppe beziehungsweise ihren Angehörigen zukommen. Als Rechtsträger sind sowohl die Volksgruppe als auch deren einzelne Angehörigen anzusehen.

Es fragt sich, ob die in der Heimat der betroffenen weißen Farmer Neuangesiedelten ein eigenes konkurrierendes Recht auf die Heimat geltend machen können. Dafür müssten sie aber auf dem Gebiet ansässig sein. Dies könnte insoweit zu bejahen sein, als auf die Zeit

Gornig, Gilbert, "Ethnische Säuberungen", Recht auf die Heimat und die Verantwortlichkeit der Vertreiber, in: AWR-Bulletin 2000, S. 19 (24).

22

Vgl. auch hierzu die Ausführungen des eidgenössischen Bundesrats, Interpellation 02.3436, v. 17.09.2002.

23

Gornig, Gilbert, (o. Fn. 21) S. 19 (21). 
vor der Landnahme durch die weißen Farmer abgehoben wird. Mit Blick auf geltendes innerstaatliches Zivilrecht in Simbabwe wird aber das wirksame Eigentumsrecht der betroffenen weißen Farmer nicht abzustreiten sein. Andernfalls bedürfte es ja auch keiner Enteignungsdekrete. Gerade unter Berücksichtigung des wirksamen Eigentumsrechts der Farmer ergibt sich, dass die notwendige Zeitspanne zur Bejahung des Merkmals „Ansässigkeit“" der Neuangesiedelten keinesfalls abgelaufen ist. Das Recht auf die Heimat kommt der weißen Volksgruppe also auch nach der Vertreibung von ihrem Land zu. Ein mittlerweile entgegenstehendes Recht der „Farmbesetzer“ besteht nicht.

\section{Afrikanische Charta der Menschenrechte und Rechte der Völker}

Kommen in Betracht auch Ansprüche aus der Afrikanischen Charta der Menschenrechte und Rechte der Völker oder Banjul-Charta ${ }^{24}$ ? Diese enthält keine Referenz auf die anderen regionalen Menschenrechtssysteme ${ }^{25}$; sie verweist vielmehr auf die Instrumente der Vereinten Nationen ${ }^{26}$. Problematisch ist hierbei die unmittelbare Anwendbarkeit der Rechte. Sie wurden nämlich als bloße Programmsätze konzipiert ${ }^{27}$. Die Charta ist allerdings - im Gegensatz zur Kulturellen Charta von Afrika - nicht nur das erste bindende Menschenrechtsinstrument, welches die verschiedenen Kategorien von Menschenrechten in einem Dokument vereint; sie schützt neben den Rechten des Einzelnen und der Völker gemäß Art. 18 Abs. 3 und 4 AfrC auch Rechte von Gruppen ${ }^{28}$.

Simbabwe ist Vertragsstaat des Abkommens.

In Betracht kommt ein Verstoß gegen das Gleichheitsgebot aus Art. 3 Abs. 1 AfrC ${ }^{29}$ aufgrund der einseitigen Konfiskations- und Vertreibungsmaßnahmen gegenüber den weißen Farmern. Durch die Enteignungsgesetzgebung Simbabwes werden faktisch ledig-

Nach ihrem Abschlußort ist das seit 21.10.1986 in Kraft gesetzte Abkommen auch „BanjulCharta“.

Wittinger, in: Jura 1999, S. 405, 410.

So Abs. 4 der Präambel, Art. 60, 62 AfrC.

27 Scherer-Leydecker, Christian, Minderheiten und sonstige ethnische Gruppen, eine Studie zur kulturellen Identität im Völkerrecht, 1997, S. 208; Oxenknecht, Renate, Der Schutz ethnischer, religiöser und sprachlicher Minderheiten in Art. 27 des Internationalen Paktes über bürgerliche und politische Rechte vom 16. Dezember 1966, 1988, S. 163; vgl. dazu auch Enonchchong, Nelson, The African Charter on Human And People's Rights: effective remedies in domestic law?, in: JAL 2002, S. 197, der auf die fehlende Durchsetzungsfähigkeit der African Commission on Human and Peoples' Rights hinweist.

Riedel, Eibe, Theorie der Menschenrechtsstandards, 1986, S. 93; Scherer-Leydecker, Christian, (o. Fn. 27) S. 207 f.

29

Text in Übersetzung, in: EuGRZ 1986, S. 677. 
lich die Angehörigen der weißen Bevölkerungsgruppe betroffen, wenn auch explizit diese Zielrichtung auf die weiße Volksgruppe im Landreformgesetz nicht erwähnt wird. Eine Ungleichbehandlung ist somit gegeben. Art. 3 AfrC enthält selbst keine Schrankenbestimmung. Fraglich ist nur, ob Art. 3 AfrC eine immanente Schranke enthält. Ferner ist durch Gesetz die gerichtliche Überprüfung von Enteignungen, welche die weißen Farmer betreffen, ausgeschlossen. Die gegenwärtigen Tendenzen der benachteiligenden Rechtsprechung der simbabwischen Gerichte gegenüber der weißen Volkgruppe zur Landreform von 2000 zeigen Ansätze einer Fortsetzung eines apartheidähnlichen Systems und damit eines neuerlich umgekehrten Minderheitenproblems ${ }^{30}$. Aufgrund des Ausschlusses der Überprüfungsmöglichkeit durch simbabwische Gerichte ist eine nicht gerechtfertigte Ungleichbehandlung durch Art. 3 Abs. 1 AfrC anzunehmen.

Durch die Vertreibung der weißen Farmer könnte auch Art. 12 Abs. 1 AfrC betroffen sein. Danach hat jedermann das Recht zur freien Wahl des Wohnsitzes. Allerdings spricht die Regelung nur von der Freiheit der Wohnsitzwahl in einem Staat. Der konkrete Wohnsitz ist somit nicht geschützt, so dass die Vertreibung von Grund und Boden - solange die Eigentümer nicht aus dem Land vertrieben werden - nicht von der Regelung erfasst ist.

Zudem wird Art. 12 Abs. 1 AfrC in S. 2 dadurch eingeschränkt, dass die gesetzlichen Vorschriften zu beachten sind. Dabei ist konsequenterweise auch auf die simbabwischen Enteignungsgesetze abzuheben.

Allerdings spricht Art. 12 Abs. 2 AfrC davon, dass jedes Individuum das Recht hat, in sein Land zurückzukehren ${ }^{31}$. Die kollektive Austreibung der weißen Volksgruppe von ihrem Land sowie die Verwehrung des Rückkehrrechts verletzt mithin dieses Recht.

Zwar gewährleistet die Charta in Art. 14 das Eigentum; jedoch folgt darauf sogleich einschränkend die Zulässigkeit von Enteignungen ${ }^{32}$. Eine Entschädigungsklausel für Enteignungen wird freilich nicht erwähnt. Dass eine Entschädigungspflicht als allgemeine Gewährleistung durch die Charta nicht vorgesehen ist, ergibt sich schon aus Art. 21 Abs. 1 und 4 AfrC. So ist gerade in Absatz 5 die Rede davon, dass afrikanische Völker in den Genuß ihrer nationalen Ressourcen kommen sollen ${ }^{33}$.

Allein Art. 21 Abs. 3 AfrC könnte ein Hinweis auf die zu beachtenden Prinzipien des Völkerrechts entnommen werden. Insofern könnte bei einer extensiven Auslegung der Charta

30

Vgl. zum System der Rassentrennung Kießwetter, Antje, Die Verfassungsentwicklung in Namibia, 1993, S. 116 f.

31 Vgl. auch Parameswaran, Benjamin, Vertreibung und Rückkehrrecht, AVR 41 2003, S. 1 (24).

32

33

Vgl. Riedel, Eibe, (o. Fn. 28) S. 95.

Riedel, Eibe, (o. Fn. 28) S. 95. 
ein völkergewohnheitsrechtlicher Rechtssatz, wonach nur unter Einschluss einer Entschädigung enteignet werden darf, auch hier zur Anwendung kommen ${ }^{34}$. Freilich sind bislang Hinweise auf eine Befolgung dieser Auslegung durch die Vertragspartner nicht ersichtlich. Art. 14 AfrC ist daher durch die Maßnahmen verletzt.

Art. 2 der AfrC statuiert ein Diskriminierungsverbot, welches auf die in der Charta explizit aufgeführten Rechte Bezug nimmt und als allgemeines Diskriminierungsverbot dahinter zurücktritt. Ein eigener Anwendungsbereich von Art. 2 AfrC ist nicht ersichtlich.

\section{Naturalrestitution statt Entschädigung}

Kommt wegen der gegenüber den Eigentümern verübten Rechtsverletzungen - Naturalrestitution oder aber Entschädigung in Betracht? Zwar gibt es grundsätzlich völkerrechtskonforme Enteignungen. Dies gilt aber nur, sofern die Enteigneten entschädigt werden. Entschädigungsleistungen auf Grundlage der Hull-Formel sind aber im südlichen Afrika bislang nicht geflossen ${ }^{35}$. Keinesfalls jedoch sind Entschädigungen auch nur annähernd adäquat geleistet worden. Gerade dies ist aber neben sofortiger und effektiver Entschädigung als Vorraussetzung in der Hull-Formel statuiert. Eine Ersatzleistung in Form der Ansiedlung auf anderem Land - wie in Südafrika, freilich auf Land minderer Qualität ${ }^{36}$, ist in Simbabwe nicht vorgesehen.

Der Herausgabe des bislang konfiszierten Privatbesitzes steht in Simbabwe im übrigen keine Unmöglichkeit entgegen. Zwar wäre auch hier an Unzumutbarkeit zu denken. Jedoch konnte aufgrund der erst kurzen neuen Besitzausübung noch kein Vertrauensschutz erworben werden, welcher das schützenswerte Interesse der Alteigentümer an der Herausgabe überwiegt.

\section{Wege der Durchsetzung von Ansprüchen}

\section{Rechtsdurchsetzung vor simbabwischen Gerichten}

Bei Verfahren eines Interessenverbandes der weißen Farmer, nämlich der Vereinigung der kommerziellen Farmer (CFU) als juristischer Person, gegen die simbabwische Regierung im Jahre 2000 wurde zunächst vom Obersten Gerichtshof die Diskriminierung der Eigentü-

Riedel, Eibe, (o. Fn. 28) S. 95.

Vgl. Mettle, Johann, The Process of Land Restitution in South Africa, in: African Journal, 1998,

36 S. 123 (138 f.).

Mettle, Johann, (o. Fn. 35) S. 123 (138). 
mer durch die Landnahme festgestellt ${ }^{37}$. Zweifelhaft ist indessen, ob es sich bei der Verfolgung der Rechtsansprüche vor simbabwischen Gerichten um einen solchen Weg handelt, der effektiven Rechtsschutz ermöglicht. Rechtsschutz in Form durchsetzbarer Titel auf Herausgabe der konfiszierten Farmen bleibt den Angehörigen der weißen Minderheit nämlich bislang versagt. Die simbabwischen Gerichte sanktionieren damit leider zum großen Teil die Politik der „Landreform“ Mugabes ${ }^{38}$. Parteifähig sind natürliche wie juristische Personen auch nach simbabwischem Recht.

Sofern die Interessenverbände der weißen Farmer mit entsprechender Legitimation ausgestattet sind, können sie die Volksgruppe repräsentieren. Sofern die Volksgruppe jedoch nicht als juristische Person auftritt, ist ihr hingegen die Parteifähigkeit versagt. Insoweit ist auch hier für eine Verfolgung der Rechte durch die Volksgruppe die angemessene Repräsentation durch ausreichende Legitimation ihrer Vertreter zu fordern.

\section{Rechtsdurchsetzung vor internationalen Institutionen}

a) Klage vor dem IGH

Für eine Klage vor dem IGH müssten die betroffenen weißen Farmer eine für das Verfahren vor dem IGH taugliche Streitpartei sein. Dabei ist die Parteifähigkeit den Staaten vorbehalten. Es gilt hier Art. 34 IGH-Statut ${ }^{39}$. Die Gruppe der weißen Farmer in Simbabwe ist jedoch lediglich eine Volksgruppe. Sie ist daher nach dem Statut vor dem Gericht nicht parteifähig.

b) Beschwerde vor dem VN-Menschenrechtsausschuss

Hätte eine Beschwerde vor dem Menschenrechtsausschuss (MRA) der Vereinten Nationen Aussicht auf Erfolg? Im Lichte der Entscheidungen des Menschenrechtsausschusses, beispielsweise zu den Anliegen der durch die damalige Tschechoslowakei enteigneten Sudetendeutschen, erscheint die Hinwendung auch der weißen Simbabwer zu diesem Organ der Vereinten Nationen durchaus erfolgversprechend, da der Ausschuss zu Fragen der Enteignung von Volksgruppenangehörigen schon in der Vergangenheit Stellung genommen hat. Der Menschenrechtsausschuss gibt allerdings lediglich ein Votum ab. Seine Entscheidungen haben eher politische Bedeutung. Eine effektive Rechtsdurchsetzung vor

Commercial Farmers Union v Minister of Lands \& Ors 2000 (2) ZLR 469, S. 489G.

38

Anders bezüglich der menschenrechtlichen Rechtsprechung der simbabwischen Gerichte zur Banjul-Charta und die Orientierung an der EGMR-Rechtsprechung freilich: Madhuku, Lovemore, (o. Fn. 2) S. 932 (934).

39

Fischer, Horst, in: Ipsen, Knut (Hrsg.): Völkerrecht, 1999, § 62 Rn. 43. 
dieser Institution scheidet somit sowohl für die Individuen als auch für die betroffene Gruppe der weißen Farmer aus.

c) Bericht an die Menschenrechtskommission

Vor einem Bericht an die Menschenrechtskommission ist zunächst der innerstaatliche Rechtsweg voll auszuschöpfen. Den weißen Farmern steht dieser Rechtsweg gegen die Enteignungen und Vertreibungen jedoch bislang nicht offen, so dass dieses Erfordernis erfüllt sein dürfte.

Das Beschwerdeverfahren wurde 1970 mit der Resolution Nr. 1503 des Wirtschafts- und Sozialrates der Vereinten Nationen geschaffen. Entsprechend dieser zeitlichen Beschränkung können nur solche Menschenrechtsverstöße vorgebracht werden, die ab 1970 erfolgt sind. Die Vertreibungen und Enteignungen gegenüber der Gruppe der weißen Farmer haben in der Hauptsache im Jahre 2000 und danach - bis heute - stattgefunden. Die Maßnahmen unterfallen damit eindeutig dem Geltungszeitraum.

Vor der Menschenrechtskommission können Verstöße gegen alle Menschenrechtskonventionen und -pakte vorgebracht werden. In diese Kategorie fallen die Vertreibungen ohne Zweifel. Notwendige Voraussetzung für das Vorbringen ist allerdings unter anderem, dass es sich um systematische Menschenrechtsverletzungen handelt. Dabei ist eine Menschenrechtsverletzung grundsätzlich dann als systematisch anzusehen, wenn wenigstens 20 Einzelfälle vorliegen oder ein Einzelfall mit mindestens 20 Betroffenen. Der erforderliche große Umfang der Maßnahmen ist mittlerweile auch hier leider unproblematisch zu bejahen, so dass die Menschenrechtsverletzung als systematisch anzusehen ist.

Maßnahmen zur Rechtsdurchsetzung der Ansprüche der weißen Farmer Simbabwes stehen der Kommission jedoch nicht zur Verfügung, so dass es bei der Feststellung der Rechtswidrigkeit bleibt.

d) Afrikanische Kommission für Menschenrechte und Rechte der Völker

Als einziges Kontrollorgan der Afrikanischen Charta der Menschenrechte und Rechte der Völker ist bis dato nur die von Art. 30 AfrC erwähnte Afrikanische Kommission für Menschenrechte und Rechte der Völker ersichtlich ${ }^{40}$. Diese ist ebenso wie die ehemalige EKMR für Staaten- und Individualbeschwerden zuständig. Dabei kommt grundsätzlich eine Mitteilung durch die geschädigten weißen Farmer nach Art. 55 der Charta in Betracht.

40

Vgl. Ipsen, Knut, (o. Fn. 39), § 50 Rn. 22; Enonchong, Nelson, (o. Fn. 28) S. 197 (214). Zum künftigen gerichtlichen Schutz nunmehr Bortfeld, Matthias, Der Afrikanische Gerichtshof für Menschenrechte, Baden-Baden, 2005, besprochen von Gärditz, VRÜ 38 (2005), S. 352. 
Allerdings ist die Afrikanische Kommission für die Menschenrechte und Rechte der Völker ebenfalls nicht zu bindenden Entscheidungen in der Lage ${ }^{41}$. Die Verletzungen der Charta, hier in Form der Verletzung der Rechte der weißen Volksgruppe, können lediglich veröffentlicht werden ${ }^{42}$. Somit ist eine effektive Durchsetzung der konkret bestehenden Ansprüche wegen der Verletzung von Rechten aus der AfrC vor der Kommission nicht möglich.

\section{Diplomatischer Schutz}

Voraussetzung für die Ausübung des Rechts auf diplomatischen Schutz ist zunächst das völkerrechtswidrige Handeln eines Drittstaats. Die Völkerrechtswidrigkeit von Vertreibung und damit einhergehender Konfiskation des Vermögens der weißen Volksgruppe auf dem simbabwischen Gebiet wurde oben festgestellt. Denn die durch den simbabwischen Staat mindestens geduldeten Handlungen verstoßen sowohl gegen Völkervertrags- als auch gegen Völkergewohnheitsrecht.

Dann müsste weiterhin eine Schutzmacht bestimmbar sein. Dies ist grundsätzlich derjenige Staat, dessen Staatsangehörigkeit die durch Maßnahmen des Aufenthaltsstaates Betroffenen haben. Zwar besitzen die weißen Farmer nicht alle die Staatsbürgerschaft desselben ausländischen Staates, so dass die Bestimmung der Schutzmacht aufgrund co-nationaler Aspekte nicht gelingt. In Betracht als Schutzmacht jener Gruppe der weißen Bevölkerung könnte indes Großbritannien kommen. Es ist nämlich aus einer historisch-faktischen Perspektive heraus der Staat zu ermitteln, der eine enge Beziehung zur betroffenen Minderheit aufweist ${ }^{43}$. Im übrigen kann mittlerweile ebenso aus der menschenrechtlichen Erweiterung des Schutzrechts abgeleitet werden, dass bei der Verletzung von Menschenrechtsnormen des Völkervertragsrechts, wie auch des Völkergewohnheitsrechts, andere Staaten ein Recht auf diplomatische Protektion im weiteren Sinne ${ }^{44}$ geltend machen ${ }^{45}$. Gerade aus dieser historischen Perspektive - mit Blick auf die Kolonialzeit - ist in Großbritannien derjenige Staat zu erkennen, welcher sich für die Belange der weißen Farmer fortgesetzt engagiert ${ }^{46}$.

Ipsen, Knut, (o. Fn. 39) § 50 Rn. 22.

Vgl. Enonchong, Nelson, (o. Fn. 28) S. 197. nungsfeld von Recht und Souveränität in Mittel- und Osteuropa, 1993, S. 128.

I.e.S. handelt es sich - konzeptionell - freilich nicht um diplomatischen Schutz, da eben nicht der Schutz eigener Staatsangehöriger bezweckt wird.

Klein, Eckart, Diplomatischer Schutz im Hinblick auf Konfiskationen deutschen Vermögens durch Polen, 1992, S. 22.

46

So hat auch Großbritannien im Zuge der ersten Übergriffe gegen die weißen Farmer im Jahre 2001 Evakuierungspläne für diese Bevölkerungsgruppe geplant. 
Danach ist Großbritannien berechtigt, diplomatischen Schutz zugunsten der weißen Bevölkerung auszuüben.

Freilich muss zuvor der innerstaatliche Rechtsweg erschöpft sein. Die Gruppe der weißen Farmer hat zunächst die simbabwischen Gerichte anzurufen. Von effektivem Rechtsschutz wird aber hier nicht die Rede sein können.

Das Recht auf Ausübung von diplomatischem Schutz kommt nach der hier vertretenen Auffassung Großbritannien als eigenes Recht zu. Weil Großbritannien aber in Simbabwe nicht primär Rechte eigener Staatsbürger, sondern vielmehr Rechte der weißen Volksgruppe vertreten würde, müsste zur Rechtskonstruktion einer Prozessstandschaft Zuflucht genommen werden. Dem allerdings kann hier nicht weiter nachgegangen werden.

\section{Fazit}

Mit dem Ende der weißen Vorherrschaft im ehemaligen Rhodesien sind offenbar Ungleichheit und Diskriminierung nicht vorbei. Die Untersuchung hat dies gezeigt am Beispiel des Ringens um das Eigentum an Grund und Boden. Die mit der Landreform durchgeführten Maßnahmen der entschädigungslosen Enteignung und Vertreibung der Angehörigen der weißen Volksgruppe in Simbabwe sind völkerrechtswidrig. Bei der weißen Bevölkerung handelt es sich um eine Volksgruppe, beziehungsweise um eine ethnische Minderheit. Die Angehörige dieser Gruppe sind sowohl als Individuen als auch als Angehörige der Minderheit durch die rechtswidrigen Handlungen adressiert. Den Angehörigen der Volksgruppe stehen die Ansprüche auf Herausgabe beziehungsweise Entschädigung nach allgemeinem Völkerrecht und das Recht auf die Heimat zu. Eine normative Kraft des Faktischen, wie sie auch das Völkerrecht kennt, konnte sich in der kurzen Zeit seit den geschilderten Ereignissen nicht herausbilden.

Leider folgte der rassistischen Diktatur der weißen Minderheit gegenwärtig eine rassistische Diktatur der schwarzen Mehrheit. Rassistisch ist das System, weil es seine Macht zu erhalten sucht durch rechtliche Mittel, die auf die Unterdrückung einer bestimmten Volksgruppe - hier der weißen Volksgruppe - abzielen ${ }^{47}$. Problematisch ist die Durchsetzung der bestehenden Rechte durch die Mitglieder der betroffenen Gruppe sowie die Gruppe als solche, da effektiver innerstaatlicher Rechtsschutz nach der lex rei sitae nicht gewährt wird. In Betracht kommt als Rechtsschutz gegen die durchgeführten Enteignungen gegenwärtig lediglich die Ausübung von diplomatischem Schutz durch Großbritannien als Schutzmacht der weißen Bevölkerung. 